\title{
Does Bleach Method Enhance Smear Positivity Of Tuberculosis Lymph Node Aspirates? A Systematic Review
}

\author{
Anju Khairwa ${ }^{1 *}$ and Kana Ram Jat ${ }^{2}$ \\ ${ }^{1}$ Department of Pathology, ESIC Model Hospital, Gurugram, India. \\ ${ }^{2}$ Departments of Pediatrics, All India Institute of Medical Sciences, New Delhi-110029, India.
}

Received: 06 February, 2018; Accepted: 29 March, 2018; Published: 2 April, 2018

*Corresponding author: Anju Khairwa, ESIC Model Hospital, Gurugram, India, Tel: +919810436138; Email: anjukhairwa@gmail.com

\begin{abstract}
Background and aims: Tubercular lymphadenitis (TBL) is most common form of extrapulmonary tuberculosis both in children and adults. Acid fast bacilli (AFB) positivity in fine needle aspiration cytology (FNAC) specimens is low; culture is time consuming and has limited availability; and newer molecular methods are costly and availability is limited. Improvement in microscopy, such as bleach method, would be a valuable tool to diagnose TBL. We aim to evaluate efficacy of bleach method to enhance smear positivity of Tuberculosis Lymph Node Aspirates.
\end{abstract}

Methods: We included studies where bleach method was compared with routine AFB staining on FNAC specimens. We searched PubMed, EMABASE and Cochrane Database Reviews. Two authors independently selected studies and extracted data for review.

Results: A total of three studies (305 patients) were included. The AFB positivity rate for routine ZN staining and bleach method was 27.0 $\%(27 / 100)$ vs $72.0 \%$ (72/1000), 33.3\% (31/93) vs $63.4 \%(59 / 93)$, and $12.5 \%(14 / 112)$ vs $60.7 \%(68 / 112)$ in three studies respectively. The studies reported that bleach method was easy, quick and less expensive. We could not combined data for pooled sensitivity and specificity as no reference standard was used in any of the included studies.

Conclusions: The limited available literature suggests that AFB positivity of FNAC specimen from TBL is higher for bleach method as compared to routine $\mathrm{ZN}$ staining. The finding is of considerable interest for resource poor countries where microscopy is only tool to diagnose TBL. Further studies are needed with proper reference standard before recommending bleach method routinely.

Key words: Bleach; Tuberculosis; lymph node; Fine needle aspiration cytology;

\section{Background}

Extrapulmonary tuberculosis comprises approximate 15 $\%$ of overall tuberculosis in HIV negative patients. Lymph node tuberculosis is most common form of extrapulmonary tuberculosis both in children and adults. Diagnosis of Tubercular Lymphadenitis (TBL) is challenge for clinicians, especially in early stages [1]. Microscopy and staining for Acid-Fast Bacilli (AFB) of Fine Needle Aspiration Cytology (FNAC) specimen is the only facility available to diagnose TBL at many centers in developing countries. Cytologic changes in FNAC specimen in TBL had wide spectrum and some of the pattern are not specific for tuberculosis $[2,3]$. Regrettably, positivity of AFB in FNAC specimens is also not good; it ranges from $9 \%$ to $46 \%$ in various studies [3]. Culture is gold standard but it is time consuming and had limited availability. Serological tests lack sensitivity and specificity and not recommended. Newer molecular methods like Polymerase Chain Reaction (PCR), GeneXpert etc. will definitely improve diagnosis of TBL but they are costly and availability is limited. Microscopy is feasible, fast, and available even at peripheral centers. If we can improve the sensitivity of AFB detection for FNAC specimens, microscopy has potential to become a valuable tool to diagnose TBL worldwide [4]. Performance of microscopy may be improved by liquefaction of clinical sample by a chemical agent followed by centrifugation before AFB staining. Bleach method is one of such technique [3]. Utility of bleach method is reported on sputum samples [5]. Isolated TBL is not uncommon in pediatric population and FNAC is most commonly used for diagnosis. There are few studies demonstrating efficacy of bleach on FNAC material but hardly any systematic review. We plan this systematic review to evaluate efficacy of bleach method to enhance smear positivity of Tuberculosis Lymph Node Aspirates.

\section{Methods}

We included studies where bleach method was used before AFB staining on FNAC specimens in TBL. Studies including both children and adults were eligible for inclusion in review. Culture was considered as reference standard. We searched PubMed (inception to $31^{\text {st }}$ May 2015), EMBASE (inception to $31^{\text {st }}$ May 2015), and Cochrane Database Reviews, April 2015. Search strategy for PubMed included ((sodium hypochlorite) OR bleach)) AND tuberculosis) AND lymph node". We searched EMBASE with key words 'Bleach'/exp OR bleach AND ('lymph'/exp OR lymph) AND node AND ('tuberculosis' / exp OR tuberculosis). References of included studies were hand searched for additional studies. 
Two authors independently selected studies and extracted data for review. Assessment of methodological quality was done using QUADAS2 [6]. We planned pooling of sensitivity and specificity by random effect method in Review manager.

\section{Results}

Pubmed, EMBASE, and Cochrane library search revealed four, five, and two results respectively. After excluding duplicates and irrelevant studies, three studies, with a total of 305 subjects, were included in the review $[7,8,9]$. Data extraction from included studies are shown in (Table 1). None of the study included culture as reference standard; therefore methodological quality of included studies was not assessed. All included studies used $5 \%$ bleach for bleach method.
All studies included both children and adults and no separate data were provided. In study by Gangane et al, diagnosis of TB was made by clinical plus FNAC findings [7]. In cases where diagnosis was difficult on FNAC findings alone, confirmation was obtained either by biopsy of the involved lymph nodes or clinical response to anti-TB therapy [7]. Chandrasekhar et al diagnosed TB by clinical plus FNAC findings. Both Gangane et al and Chandrasekhar et al, reported six pattern of FNAC findings [7,9] (Table 1). Pattern 1, 3, 4 in FNAC were considered quite specific for tuberculosis. In all three included studies, AFB positivity increased markedly after modified bleach method (Table 1 ). The AFB positivity rate for routine ZN staining and modified bleach method was $27.0 \%$ (27/100) vs $72.0 \%$ (72/1000), 33.3\% (31/93) vs 63.4\% (59/93), and $12.5 \%(14 / 112)$ vs $60.7 \%(68 / 112)$ in study by Gangane et

Table 1: Data extraction from included studies

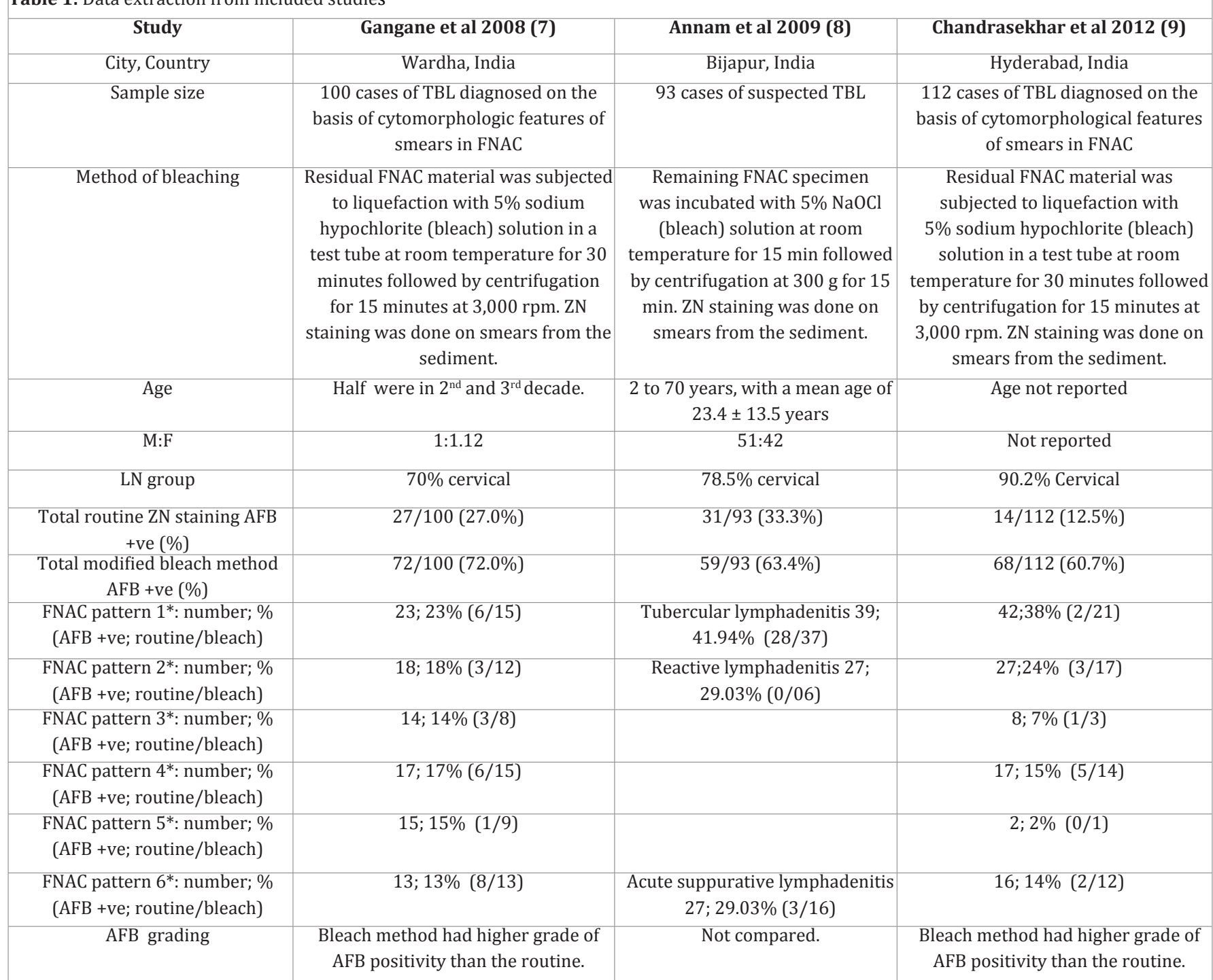

TBL: Tuberculous lymphadenitis, FNAC: fine needle aspiration cytology, *Pattern1: Epithelioid granulomas with Langerhans giant cells and caseous necrosis, Pattern 2: Numerous clusters of epithelioid cells in a reactive background, Pattern 3: Mostly caseous necrotic material with few epithelioid cells, Pattern 4: Caseous necrotic material with few lymphocytes and histiocytes; no epithelioid cells, Pattern 5: Only caseous necrosis without any other cell types, Pattern 6: Tuberculous abscess showing predominantly neutrophils along with degenerating epithelioid cells and semifluid necrotic material. 
al, Annam et al, Chandrasekhar et al, respectively [7, 8, 9]. In any of the study, the modified bleach method did not miss any case of AFB positivity that was detected on routine $\mathrm{ZN}$ staining. There was also AFB positivity by bleach method among cases that reported as reactive or supportive lymphadenitis on FNAC and these cases responded well to ant-TB therapy [8]. A total of eight $(8.6 \%)$ and $16(14.3 \%)$ patients were HIV positive in study by Annam et al and Chandrasekhar et al respectively [8, 9]. AFB was positive by routine and bleach method in four $(25 \%)$ and $14(87.5 \%)$ patients respectively among HIV positive patients in study by Chandrasekhar, et al [9]. The studies reported that bacilli were seen with ease with better preserved morphology in clear background after bleach method which resulted in less strain on eyes and reduced time for slide examination $[7,9]$.

We could not combined data for pooled sensitivity and specificity as no reference standard was used in any of the included studies.

\section{Discussion}

Though none of the included study used reference standard, AFB positivity was markedly high for modified bleach method as compared to routine ZN staining in FNAC specimens from suspected TBL patients.

The need for an improvised, low-cost microscopy technique for diagnosis of TBL both in children and adults cannot be overemphasized. Bleach method is may be such a method. Khubnani et al, studied 55 cases of extrapulmonary TB (18 aspirates from body fluids, 18 from abscesses drained from various body sites, 17 from lymph nodes and two from skin scrapings) with cytology, routine ZN staining and bleach method and found that cytology suggested TB in $43.36 \%$ cases and AFB positivity was $21.8 \%$ and $70.9 \%$ by routine $\mathrm{ZN}$ staining and bleach method respectively [3]. A few reviews had reported better sensitivity of bleach method as compared to routine ZN staining for sputum examination [5, 10]. Cattamanchi et al, reported higher pooled sensitivity for bleach centrifugation $(65 \%, 95 \%$ CI $59 \%$ to $71 \%$, I2 $=75 \%$ ) than for direct microscopy (56\%, 95\% CI $49 \%$ to $63 \%$, I $2=87 \%$ ) for sputum examination [5]. In the same review, pooled specificity was high for both bleach centrifugation microscopy (96\%, 95\% CI, 93\% to 98\%, I2=88\%) and direct microscopy (98\%, 95\% CI $97 \%$ to $99 \%, \mathrm{I} 2=46 \%$ ) which is obvious (5). The present review reported higher AFB positivity of bleach method as compared to routine $\mathrm{ZN}$ staining consistently in all included studies for FNAC specimen in TBL.

Laboratory Acquired Infection (LAI) with Mycobacterium tuberculosis is a concern worldwide as yearly incidence is reported to be about 0.3 per 1000 people [11]. Another advantage of bleach method is decrease risk of LAI among laboratory staff as $\mathrm{NaOCl}$ kills the mycobacterium. But, it is also a disadvantage that processed sample cannot be used for culture.

The improved AFB positivity by bleach method may be due to changes in the surface properties of the mycobacterium (i.e. charge and hydrophobicity) and/or denaturation of the material by $\mathrm{NaOCl}$ that leads to flocculation and subsequent increased sedimentation rate of the bacilli $[8,12]$. Centrifugation increases density of bacilli per microscopic field and bleach also reduces debris resulting in clear background [13].

The discrepancy between cytological diagnosis and AFB positivity by bleach method was mostly for reactive lymphadenitis and acute suppurative lymphadenitis in FNAC. It may be due to loss of sparse epithelioid cells in dense population of polymorphous lymphoid cells in reactive lymphadenitis and due to loss of bacilli in dense necrotic material in acute suppurative lymphadenitis.

Strength of the review includes broad search strategy and selection of study and extraction of data by two authors independently. There are limitations of review also. The literature on review topic is limited. The included studies are of poor quality. None of the included studies had reference standard test. Data could not be pooled because lack of sufficient data. More robust diagnostic accuracy studies are needed for this underutilized, potentially beneficial test especially for resource poor settings.

\section{Conclusion}

The limited available literature suggests that AFB positivity of FNAC specimen from tubercular lymphadenitis may be improved markedly by liquefaction of aspirated material with bleach followed by centrifugation. This finding is of considerable interest for resource poor countries where tubercular lymphadenitis is still constitute a significant load of tuberculosis in community. Further studies are needed with proper reference standard test before recommending bleach method routinely.

\section{References}

1. Seth V, Kabra SK, Jain Y, Semwal OP, Mukhopadhyaya S and Jensen RL. Tubercular lymphadenitis: Clinical manifestations. Indian J Pediatr. 1995;62(5):565-570.

2. Das DK, Pant JN, Chachra KL, Murthy NS, Satyanarayan L and Thankamma TC, et al. Tuberculous lymphadenitis: correlation of cellular components and necrosis in lymph-node aspirate with A.F.B. positivity and bacillary count. Indian J Pathol Microbiol. 1990;33(1):1-10.

3. Khubnani $\mathrm{H}$ and Munjal K. Application of bleach method in diagnosis of extra-pulmonary tuberculosis. Indian J Pathol Microbiol. 2005;48(4):546-50.

4. Angeby K A, Hoffner SE and Diwan VK. Should the "bleach microscopy method" be recommended for improved case detection of tuberculosis? Literature review and key person analysis. Int J Tuberc. Lung Dis. 2004;8(7):806-815.

5. Cattamanchi A, Davis J, Pai M, Huang L, Hopewell P and Steingart K. Does bleach processing increase the accuracy of sputum smear microscopy for diagnosing pulmonary tuberculosis? J Clin Microbiol. 2010;48(7):2433-2439.

6. Whiting PF, Rutjes AWS, Westwood ME, Mallett S, Deeks JJ and Reitsma JB, et al. QUADAS-2: a revised tool for the quality assessment of diagnostic accuracy studies. Ann Intern Med. 2011;155(8):529-536. 
7. Gangane N, Anshu and Singh R. Role of modified bleach method in staining of acid-fast bacilli in lymph node aspirates. Acta Cytol. 2008;52(3):325-328.

8. Annam V, Karigoudar MH and Yelikar BR. Improved microscopical detection of acid-fast bacilli by the modified bleach method in lymphnode aspirates. Indian J Pathol Microbiol. 2009;52(3):349-352.

9. Chandrasekhar B and Prayaga AK. Utility of concentration method by modified bleach technique for the demonstration of acid-fast bacilli in the diagnosis of tuberculous lymphadenopathy. J Cytol Indian Acad Cytol. 2012;29(3):165-168.

10. Srikanth P, Kamesh S and Daley P. Bleach optimization of sputum smear microscopy for pulmonary tuberculosis. Indian J Tuberc. 2009;56(4):174-184.
11. Singh K. Laboratory-acquired infections. Clin Infect Dis Off Publ Infect Dis Soc Am. 2009;49(1):142-147.

12. Gebre N, Karlsson U, Jönsson G, Macaden R, Wolde A and Assefa A, et al. Improved microscopical diagnosis of pulmonary tuberculosis in developing countries. Trans R Soc Trop Med Hyg. 1995;89(2):191193.

13. Miörner H, Ganlöv G, Yohannes Z and Adane Y. Improved sensitivity of direct microscopy for acid-fast bacilli: sedimentation as an alternative to centrifugation for concentration of tubercle bacilli. J Clin Microbiol. 1996;34(12):3206-3207. 